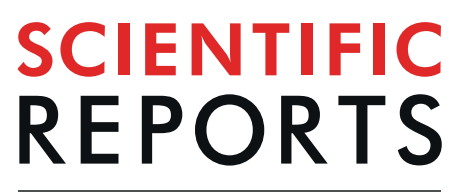

natureresearch

\title{
Association between Coronary Artery Measurements and Retinal Microvasculature in Children with New Onset of Kawasaki Disease
}

\begin{abstract}
Edward Jianyang Lim¹, Izzuddin M. Aris ${ }^{2,3,4}$, Jonathan Choo ${ }^{5}$, Tien Yin Wong ${ }^{6}$ \& Ling-Jun Li $\mathbb{C}^{6,7,8 *}$
About a quarter of children with new onset of Kawasaki disease (KD) encounter coronary arterial involvement. While KD is known to cause vasculitis of medium-sized vessels, few studies have been done to study the involvement of the microcirculation. We aimed to investigate the association between coronary arterial dilatation and retinal microvasculature in a pilot setting, in order to further study the pathophysiological mechanism of KD from the perspective of small vessels changes. We performed a cross-sectional, observational, hospital-based study on 11 children aged 2 years and above with new-onset KD. Cardiac imaging technicians performed the echocardiographic examinations and recorded right coronary artery (RCA), left coronary artery (LCA) and left anterior descending artery (LAD). Qualified retinal graders reviewed and graded standardised retinal photographs to assess retinal microvascular parameters. Among 11 participants, there were 7 boys and 4 girls. Median and interquartile range of participants' age were 5.92 (3.08) years. After adjusting for age and sex, each unit increase in LAD ( $\mathrm{mm}$ ) was significantly associated with increment of retinal arteriolar tortuosity $\left(4.25 \times 10^{-5}\right.$ units, $95 \%$ Confidence Interval: $\left.1.19,7.32\right)$. Retinal arteriolar geometric changes were associated with LAD dilatation in 11 children with new onset of KD. Our pilot provided proof-of-concept that retinal imaging might be useful for detecting coronary arterial involvement in young children with $\mathrm{KD}$ and it needs further investigation.
\end{abstract}

Kawasaki Disease (KD) is a childhood vasculitis that has become increasingly common in developed countries ${ }^{1}$. Although the acute inflammatory phase is self-limited, cardiac complications may develop and these cause significant long term morbidity and mortality in $20-25 \%$ of untreated paediatric patients ${ }^{2}$. Early diagnosis and treatment is crucial for KD patients, in order to prevent mortality that peaks 15 to 45 days after the initial onset of fever ${ }^{3}$. The pathophysiology of KD arteriopathy has been widely speculated to be characterised by 3 linked processes of necrotising arteritis, subacute/chronic vasculitis and luminal myofibroblastic proliferation. While KD is known to cause vasculitis of medium-sized vessels, few studies have been done to study the involvement of the microcirculation. Therefore, there is a need to further study pathophysiological mechanism of KD from the perspective of small vessel changes. As such, examining retinal vasculature in a non-invasive manner may enhance our ability to explore KD pathogenesis via microcirculation among paediatric patients with new-onset KD.

Retinal imaging technology has enabled researchers to assess changes in retinal microvascular morphology (e.g. vascular calibre, curvature tortuosity, fractal dimension and branching angle) in a non-invasive and accurate manner. Such systematic parameters mirror an "optimal state" of the retinal microcirculation, which may also reflect systemic microcirculation ${ }^{4}$. A previous cross-sectional study conducted on Singaporean and Australian chronic paediatric KD patients, who suffered relapses and were treated for years, found that wider retinal venules

\footnotetext{
${ }^{1}$ Yong Loo Lin School of Medicine, National University of Singapore, Singapore, Singapore. ${ }^{2}$ Singapore Institute for Clinical Sciences, Agency for Science, Technology and Research, Singapore, Singapore. ${ }^{3}$ Department of O\&G, Yong Loo Lin School of Medicine, National University of Singapore, Singapore, Singapore. ${ }^{4}$ Division of Chronic Disease Research Across the Lifecourse, Department of Population Medicine, Harvard Medical School and Harvard Pilgrim Health Care Institute, Boston, Massachusetts, USA. ${ }^{5}$ Cardiology Service, KK Women's and Children's Hospital, Singapore, Singapore. ${ }^{6}$ Singapore Eye Research Institute, Singapore National Eye Centre, Singapore, Singapore. ${ }^{7}$ OBGYN Academic Clinician Program (ACP), Duke-NUS Medical School, Singapore, Singapore. ${ }^{8}$ Division of O\&G, KK Women's and Children's Hospital, Singapore, Singapore. *email: obgllj@nus.edu.sg
} 
were associated with coronary artery abnormalities, such as giant coronary artery aneurysms ${ }^{5}$. However, it is still unknown whether such retinal vascular changes exist during the new-onset other than recurrent acute phase of $\mathrm{KD}$. If such proof-of-concept is proven, it is worth exploring the potential screening value of retinal technology for coronary arterial dilation detection among young children with new-onset $\mathrm{KD}$, in addition to conventional screening techniques.

In this pilot study, we examined the association of cardiac measures and retinal microvasculature in 11 young children with new-onset KD. We hypothesised that early dilatation of coronary arteries is associated with a series of changes in retinal microvasculature (e.g. retinal arteriolar dilation), and it would be worth exploring further in terms of the screening value of retinal imaging among children at risk of coronary arterial involvement with new-onset KD.

\section{Methods}

In order to minimise the possibility of unintentionally sharing information that can be used to re-identify private information, the data and study materials that support the findings of this study will not be available. This is a cross-sectional, observational, hospital-based study on new-onset of paediatric KD patients. We recruited patients during their first ward admission before intravenous immunoglobulin (IVIG) treatment was given, at the KK Women's and Children's Hospital, Singapore. We considered all children eligible for the study if they were: (1) aged 2 years and above; (2) had newly diagnosed and untreated KD; and (3) without other comorbidities during the period of assessment. We excluded patients who were suffering from any active eye diseases (e.g. keratitis) except for refractive error. We screened 14 patients from May 2015 to July 2016. A total of 11 patients (median 5.92 years, inter-quartile range 3.08 years) were included for final analysis (response rate $78.6 \%$ ) after excluding three patients [ 2 declined participation and 1 with ungradable retinal photos]. We conducted the study according to the tenets of the Declaration of Helsinki, and obtained approval from the SingHealth Centralized Institutional Review Board and the National Health Group's Domain Specific Review Board. Trained research coordinators obtained written informed consents from the legal guardians of all paediatric patients.

Cardiac imaging technicians performed the echocardiographic examinations and recorded cardiac measures such as right coronary artery (RCA), left coronary artery (LCA) and left anterior descending artery (LAD). Cardiologists reviewed all scans to ensure the accuracy of the cardiac measures. We calculated z-scores of RCA, LCA and LAD based on the recently published guidelines in a large series of US children with all races and ethnicities $(\mathrm{n}=3566)$, using the equation $\mathrm{z}=\left[\left(\mathrm{x} / \mathrm{BSA}^{\alpha}\right)-\mu\right] / \sigma$, where $\mathrm{x}$ is the observed parameter value, BSA is the body surface area, $\alpha$ is the exponential power to be used with BSA, $\mu$ is the mean and $\sigma$ is the standard deviation ${ }^{6}$. The BSA measurements were derived using the Haycock Formula, BSA $\left(\mathrm{m}^{2}\right)=0.024265 \times$ height $(\mathrm{cm})^{0.3964} \times$ weight $(\mathrm{kg})^{0.53787}$. We also collected systolic blood pressure (SBP) and anthropometric measures to calculate body mass index (BMI). The SBP z-scores were calculated based on the very large database from fourth report from the National High Blood Pressure Education Program Working Group on Children and Adolescents $(n=63,227)$, using the equation $z=(x-\mu) / \sigma$, where $x$ is the observed parameter value, $\mu$ is the mean and $\sigma$ is a given value from the appendix table in the repor $\mathrm{t}^{8}$. The BMI $\mathrm{z}$-scores were calculated based on the United States Growth Charts, National Center for Health Statistics, using the equation $\mathrm{z}=\left[(\mathrm{x} / \mathrm{M})^{* *} \mathrm{~L}-1\right] / \mathrm{LS}, \mathrm{L} \neq 0$ or $\mathrm{z}=\ln$ $(\mathrm{x} / \mathrm{M}) / \mathrm{S}, \mathrm{L}=0$, where $\mathrm{x}$ is the observed parameter value, $\mathrm{L}, \mathrm{M}$ and $\mathrm{S}$ are the values from the appropriate table corresponding to the age in months of the child and $(\mathrm{x} / \mathrm{M})^{* *} \mathrm{~L}$ is raising the quantity $(\mathrm{X} / \mathrm{M})$ to the Lth power ${ }^{9}$.

We took retinal photographs within the same day of definite KD diagnosis and yet before IVIG administration. However, if the KD diagnosis had been unclear via ECHO exam for the first time and the patient was temporarily diagnosed as incomplete $\mathrm{KD}$ (defined as children are suspected of having KD who do not fulfil diagnostic criteria), IVIG would still be given by the paediatric cardiologists, in order to prevent the patients from developing other manifestations subsequently. And therefore, we performed retinal photography 1-2 days after a re-examination on ECHO with a definite diagnosis and yet after IVIG treatment. Of the 11 subjects, 5 subjects received IVIG treatment before retinal photographs were taken. We used a non-mydriatic retinal camera (CR-DGi, Canon, Tokyo, Japan) to obtain one optic nerve centre and one macula centre photo for each eye, without any pupil dilation. Qualified retinal graders reviewed and graded retinal photographs using a semi-automated software (Singapore I Vessel Analyser [SIVA], version 4.0, Singapore Eye Research Institute, Singapore), which assessed retinal microvascular parameters including calibre, branching angle, fractal dimension and tortuosity, according to standardized protocols ${ }^{10}$ (Supplementary Figure 1). We assessed intra-grader reliability using $10 \%(\mathrm{n}=2)$ of randomly selected retinal photographs from our study, and the intra-class correlation coefficient was above 0.80 for all retinal vascular measures.

We examined the association between coronary arterial measures and retinal vascular measures using linear regression in two models: Model 1, unadjusted and Model 2, adjusted for child age and sex. We used SPSS 19.0 (IBM Analytics, Chicago, US) to conduct all statistical analyses. A $95 \%$ confidence interval (CI) and a $5 \%$ level of significance were utilised; therefore, we took statistical significance for the $\mathrm{p}$ value to be less than 0.05 for the two-tailed test.

\section{Results}

Among 11 patients, majority were males (63.6\%) and of Chinese ethnicity (81.8\%). There were no sex differences in all of the measures. Table 1 shows other characteristics of the patients.

Tables 2 and 3 show the associations between coronary artery parameters and retinal vessel parameters in patients with new-onset KD. In the unadjusted model, each $1.0 \mathrm{~mm}$ increase in RCA was significantly associated with increased retinal arteriolar fractal dimension $\left(0.11 \mathrm{Df} ; 95 \% \mathrm{CI}: 0.33 \times 10^{-3}, 0.22\right)$. Each $1.0 \mathrm{~mm}$ increase in LAD was significantly associated with increment of retinal arteriolar branching angle $(11.46$ degrees; $0.77,22.16)$ and retinal arteriolar curvature tortuosity $\left(4.78 \times 10^{-5}\right.$ units; $\left.2.30,7.27\right)$ (Table 2). Furthermore, each unit increase in LAD z-score was significantly associated with increased retinal arteriolar fractal dimension $\left(0.06 \mathrm{Df} ; 0.39 \times 10^{-3}\right.$, 0.11 ) (Table 3). However, after adjusting for age and sex, only the association between LAD and retinal arteriolar tortuosity remained significant $\left(4.25 \times 10^{-5}\right.$ units, $95 \%$ CI: $\left.1.19,7.32\right)$ (Table 2$)$. We performed a sensitivity analysis additionally adjusting for IVIG treatment (yes vs. no), which did not change our estimates significantly. 


\begin{tabular}{|c|c|c|c|}
\hline \multirow[b]{2}{*}{ Pre-IVIG Characteristics } & All $(n=11)$ & Male $(n=7)$ & Female $(n=4)$ \\
\hline & $\begin{array}{l}\text { Median (IQR) } \\
\text { or n (\%) }\end{array}$ & $\begin{array}{l}\text { Median (IQR) } \\
\text { or n }(\%)\end{array}$ & $\begin{array}{l}\text { Median (IQR) } \\
\text { or n }(\%)\end{array}$ \\
\hline Age & $5.92(3.08)$ & $6.17(3.08)$ & $4.46(2.58)$ \\
\hline \multicolumn{4}{|l|}{$\underline{\operatorname{Sex}}$} \\
\hline Male & $7(63.6 \%)$ & - & - \\
\hline \multicolumn{4}{|l|}{ Race/Ethnicity } \\
\hline Chinese & $9(81.8 \%)$ & $6(54.5 \%)$ & $3(27.3 \%)$ \\
\hline Malay & $1(9.1 \%)$ & $1(9.1 \%)$ & $0(0 \%)$ \\
\hline Caucasian & $1(9.1 \%)$ & $0(0 \%)$ & $1(9.1 \%)$ \\
\hline \multicolumn{4}{|l|}{ Blood Pressure and Anthropometry } \\
\hline $\mathrm{SBP}, \mathrm{mmHg}$ & $98.0(23.0)$ & $99.0(31.0)$ & $94.0(21.5)$ \\
\hline SBP z-score & $1.03(2.10)$ & $1.15(2.88)$ & $0.51(1.80)$ \\
\hline BMI, $\mathrm{kg} / \mathrm{m}^{2}$ & $14.7(2.6)$ & $13.9(2.7)$ & $15.8(2.1)$ \\
\hline BMI z-score & $-0.46(2.91)$ & $-1.38(2.67)$ & $0.21(1.46)$ \\
\hline $\mathrm{BSA}, \mathrm{m}^{2}$ & $0.69(0.20)$ & $0.73(0.15)$ & $0.64(0.14)$ \\
\hline \multicolumn{4}{|l|}{ Laboratory Markers } \\
\hline ESR, $\mathrm{mm} / \mathrm{h}$ & $96.0(34.0)$ & $74.0(37.0)$ & $99.5(15.3)$ \\
\hline CRP, mg/L & $68.5(94.8)$ & $68.5(54.7)$ & $77.2(153.8)$ \\
\hline $\mathrm{WBC}, \mathrm{x} 10^{9} / \mathrm{L}$ & $14.7(5.2)$ & $15.5(10.5)$ & $12.0(2.9)$ \\
\hline $\mathrm{Hb}, \mathrm{g} / \mathrm{dL}$ & $11.0(1.6)$ & $11.2(1.3)$ & $10.6(1.9)$ \\
\hline \multicolumn{4}{|l|}{ Echo Measures } \\
\hline $\mathrm{RCA}, \mathrm{mm}$ & $1.77(0.41)$ & $1.77(0.36)$ & $1.77(0.73)$ \\
\hline RCA z-score & $-0.30(1.06)$ & $-0.30(1.06)$ & $-0.32(1.25)$ \\
\hline LCA, mm & $2.26(0.37)$ & $2.26(0.29)$ & $2.35(0.40)$ \\
\hline LCA z-score & $-0.48(0.89)$ & $-0.69(1.27)$ & $-0.06(0.44)$ \\
\hline $\mathrm{LAD}, \mathrm{mm}$ & $1.78(0.32)$ & $1.82(0.25)$ & $1.62(0.48)$ \\
\hline LAD z-score & $0.43(0.78)$ & $0.43(0.62)$ & $0.24(1.32)$ \\
\hline \multicolumn{4}{|l|}{ Retinal Vessels Parameters } \\
\hline Arteriolar diameter, $\mu \mathrm{m}$ & $127.62(9.60)$ & $127.62(9.60)$ & $126.76(23.00)$ \\
\hline Venular diameter, $\mu \mathrm{m}$ & $182.47(15.01)$ & $179.24(17.41)$ & $187.04(12.28)$ \\
\hline Arteriolar fractal dimension, $\mathrm{Df}$ & $1.22(0.067)$ & $1.22(0.067)$ & $1.22(0.12)$ \\
\hline Venular fractal dimension, Df & $1.21(0.050)$ & $1.21(0.053)$ & $1.20(0.13)$ \\
\hline Arteriolar curvature tortuosity, unit $\left({ }^{-5}\right)$ & $13.86(5.20)$ & $15.26(5.89)$ & $10.70(3.58)$ \\
\hline Venular curvature tortuosity, unit $\left(\left(^{-5}\right)\right.$ & $12.44(2.22)$ & $11.90(2.93)$ & $12.64(3.42)$ \\
\hline Arteriolar branching angle, degree & $86.93(10.75)$ & $90.93(16.92)$ & $85.58(7.88)$ \\
\hline Venular branching angle, degree & $82.95(19.99)$ & $82.28(16.52)$ & $86.01(17.84)$ \\
\hline
\end{tabular}

Table 1. Characteristics of $11 \mathrm{KD}$ paediatric patients in our study. Abbreviations: IVIG, Intravenous immunoglobulin; SBP, Systolic blood pressure; BMI, Body mass index; BSA, Body surface area; ESR, Erythrocyte sedimentation rate; CRP, C-reactive protein; WBC, White blood cell; Hb, Haemoglobin; RCA, Right coronary artery; LCA, Left coronary artery; LAD, Left anterior descending artery.

\section{Discussion}

In this hospital-based, cross-sectional study among children with new-onset of $\mathrm{KD}$, a larger left anterior descending artery index was associated with greater values in retinal arteriolar geometric parameters especially curvature tortuosity.

A previous study found that increment of coronary artery diameters was associated with wider retinal venules in paediatric KD patients with recurrent acute flares ${ }^{5}$. We found however, that retinal arteriolar changes, instead of retinal venular changes, were associated with coronary artery changes during the acute phase of new KD onset. We speculated that the retinal venular changes in the published study might be a result of general upregulated systemic inflammation ${ }^{1}$, instead of specific features of retinal vascular morphology related to KD. In our study, we reported a series of changes in retinal arteriolar geometric features including greater fractal dimension and curvature tortuosity. As suggested in previous studies, fractal dimension measures the degree of geometric complexity $^{11}$, while tortuosity assesses the shape of retinal vessel ${ }^{12}$. Both geometric parameters reflect an optimal blood circulation in the retina. Thus, altered fractal dimension and/or curvature tortuosity could reflect increased angiogenesis or oxidative stress in response to inflammatory-induced neuro-retinal hypoxia and endothelial dysfunction ${ }^{5}$. Although increased retinal tortuosity has been speculated to be related to increased blood flow and angiogenesis ${ }^{13-15}$, the true physiologic significance of increased retinal arteriolar tortuosity is still not fully understood. Aside from inflammation and systemic vascular conditions, other causes of increased retinal arteriolar tortuosity include established cardiovascular risk factors including increased age, BMI, BP triglyceride levels, total and low-density lipoprotein cholesterol ${ }^{12,16}$. Due to inflammatory-mediated mechanisms in KD coronary 


\begin{tabular}{|c|c|c|c|c|}
\hline \multirow{2}{*}{\multicolumn{2}{|c|}{$\begin{array}{l}\text { Retinal microvascular } \\
\text { measures }\end{array}$}} & \multicolumn{3}{|l|}{ Echo Measures } \\
\hline & & \multirow{2}{*}{\begin{tabular}{|l|} 
RCA \\
per $1 \mathrm{~mm} \uparrow$ \\
\end{tabular}} & \multirow[t]{2}{*}{$\begin{array}{l}\text { LCA } \\
\text { per } 1 \mathrm{~mm} \uparrow\end{array}$} & \multirow[t]{2}{*}{$\begin{array}{l}\text { LAD } \\
\text { per } 1 \mathrm{~mm} \uparrow\end{array}$} \\
\hline \multicolumn{2}{|c|}{ Retinal Arteriolar Diameter, $\mu \mathrm{m}$} & & & \\
\hline Model 1 & $\begin{array}{l}\beta, 95 \% \mathrm{CI} \\
\text { P value }\end{array}$ & $\begin{array}{l}1.61(-24.20,27.41) \\
0.89\end{array}$ & $\begin{array}{l}-4.81(-22.88,13.27) \\
0.56\end{array}$ & $\begin{array}{l}-0.36(-15.43,14.71) \\
0.96\end{array}$ \\
\hline Model 2 & \begin{tabular}{|l|}
$\beta, 95 \% \mathrm{CI}$ \\
P value
\end{tabular} & $\begin{array}{l}2.60(-30.22,35.43) \\
0.86\end{array}$ & $\begin{array}{l}-4.92(-26.23,16.39) \\
0.60\end{array}$ & $\begin{array}{l}0.86(-20.02,21.74) \\
0.93\end{array}$ \\
\hline \multicolumn{5}{|c|}{ Retinal Venular Diameter, $\mu \mathrm{m}$} \\
\hline Model 1 & $\begin{array}{l}\beta, 95 \% \mathrm{CI} \\
\text { P value } \\
\end{array}$ & $\begin{array}{l}-10.27(-41.16,20.63) \\
0.47\end{array}$ & $\begin{array}{l}-8.61(-30.39,13.17) \\
0.40\end{array}$ & $\begin{array}{l}2.46(-16.03,20.95) \\
0.77\end{array}$ \\
\hline Model 2 & $\begin{array}{l}\beta, 95 \% \mathrm{CI} \\
\text { P value }\end{array}$ & $\begin{array}{l}-10.34(-50.00,29.32) \\
0.56\end{array}$ & $\begin{array}{l}-8.41(-34.27,17.45) \\
0.47\end{array}$ & $\begin{array}{l}2.08(-23.71,27.86) \\
0.85\end{array}$ \\
\hline \multicolumn{5}{|c|}{$\underline{\text { Retinal Arteriolar Fractal Dimension, Df }}$} \\
\hline Model 1 & $\begin{array}{l}\beta, 95 \% \mathrm{CI} \\
\text { P value } \\
\end{array}$ & $\begin{array}{l}0.11\left(0.33 \times 10^{-3}, 0.22\right) \\
0.05\end{array}$ & $\begin{array}{l}-0.30 \times 10^{-2}(-0.10,0.09) \\
0.95\end{array}$ & $\begin{array}{l}0.03(-0.05,0.11) \\
0.39\end{array}$ \\
\hline Model 2 & $\begin{array}{l}\beta, 95 \% \mathrm{CI} \\
\text { P value }\end{array}$ & $\begin{array}{l}0.11(-0.03,0.25) \\
0.09\end{array}$ & $\begin{array}{l}-0.40 \times 10^{-2}(-0.12,0.11) \\
0.94\end{array}$ & $\begin{array}{l}0.04(-0.06,0.15) \\
0.35\end{array}$ \\
\hline \multicolumn{5}{|c|}{ Retinal Venular Fractal Dimension, Df } \\
\hline Model 1 & $\begin{array}{l}\beta, 95 \% \mathrm{CI} \\
\text { P value }\end{array}$ & $\begin{array}{l}0.05(-0.08,0.18) \\
0.38\end{array}$ & $\begin{array}{l}-0.33(-0.12,0.06) \\
0.42\end{array}$ & $\begin{array}{l}-0.02(-0.09,0.06) \\
0.59\end{array}$ \\
\hline Model 2 & $\begin{array}{l}\beta, 95 \% \mathrm{CI} \\
\text { P value }\end{array}$ & $\begin{array}{l}0.03(-0.12,0.19) \\
0.64\end{array}$ & $\begin{array}{l}-0.04(-0.13,0.06) \\
0.41\end{array}$ & $\begin{array}{l}-0.02(-0.12,0.07) \\
0.57\end{array}$ \\
\hline \multicolumn{5}{|c|}{ Retinal Arteriolar Branching Angle, degree } \\
\hline Model 1 & $\begin{array}{l}\beta, 95 \% \mathrm{CI} \\
\text { P value }\end{array}$ & $\begin{array}{l}2.73(-20.74,26.21) \\
0.80\end{array}$ & $\begin{array}{l}3.71(-12.88,20.29) \\
0.63\end{array}$ & $\begin{array}{l}11.46(0.77,22.16) \\
0.04\end{array}$ \\
\hline Model 2 & $\begin{array}{l}\beta, 95 \% \mathrm{CI} \\
\text { P value }\end{array}$ & $\begin{array}{l}8.17(-18.05,34.39) \\
0.49\end{array}$ & $\begin{array}{l}4.66(-12.86,22.17) \\
0.55\end{array}$ & $\begin{array}{l}10.79(-3.55,25.13) \\
0.12\end{array}$ \\
\hline \multicolumn{5}{|c|}{ Retinal Venular Branching Angle, degree } \\
\hline Model 1 & $\begin{array}{l}\beta, 95 \% \mathrm{CI} \\
\text { P value }\end{array}$ & $\begin{array}{l}1.17(-26.71,29.05) \\
0.93\end{array}$ & $\begin{array}{l}-4.15(-23.81,15.51) \\
0.64\end{array}$ & $\begin{array}{l}3.05(-13.06,19.16) \\
0.68\end{array}$ \\
\hline Model 2 & $\begin{array}{l}\beta, 95 \% \mathrm{CI} \\
\text { P value }\end{array}$ & $\begin{array}{l}2.89(-31.71,37.49) \\
0.85\end{array}$ & $\begin{array}{l}-4.33(-26.94,18.29) \\
0.67\end{array}$ & $\begin{array}{l}7.05(-14.06,28.15) \\
0.46\end{array}$ \\
\hline \multicolumn{5}{|c|}{$\underline{\text { Retinal Arteriolar Curvature Tortuosity, unit }} \stackrel{-5}{-}$} \\
\hline Model 1 & $\begin{array}{l}\beta, 95 \% \mathrm{CI} \\
\text { P value } \\
\end{array}$ & $\begin{array}{l}1.01(-6.45,8.48) \\
0.77\end{array}$ & $\begin{array}{l}1.84(-3.33,7.02) \\
0.44\end{array}$ & $\begin{array}{l}4.78(2.30,7.27) \\
0.002\end{array}$ \\
\hline Model 2 & $\begin{array}{l}\beta, 95 \% \mathrm{CI} \\
\text { P value }\end{array}$ & $\begin{array}{l}1.31(-6.29,8.91) \\
0.70\end{array}$ & $\begin{array}{l}2.10(-2.63,6.83) \\
0.33\end{array}$ & $\begin{array}{l}4.25(1.19,7.32) \\
0.01\end{array}$ \\
\hline \multicolumn{5}{|c|}{$\underline{\text { Retinal Venular Curvature Tortuosity, unit }}{ }^{-5}$} \\
\hline Model 1 & $\begin{array}{l}\beta, 95 \% \mathrm{CI} \\
\text { P value }\end{array}$ & $\begin{array}{l}-0.08(-5.47,5.32) \\
0.98\end{array}$ & $\begin{array}{l}0.51(-3.33,4.34) \\
0.77\end{array}$ & $\begin{array}{l}1.92(-0.88,4.72) \\
0.16\end{array}$ \\
\hline Model 2 & $\begin{array}{l}\beta, 95 \% \mathrm{CI} \\
\text { P value }\end{array}$ & $\begin{array}{l}1.73(-3.49,6.94) \\
0.46\end{array}$ & $\begin{array}{l}0.80(-2.72,4.33) \\
0.61\end{array}$ & $\begin{array}{l}1.22(-2.06,4.49) \\
0.41\end{array}$ \\
\hline
\end{tabular}

Table 2. Association between coronary artery parameters and retinal vessel parameters in patients with new onset of Kawasaki disease. Abbreviations: RCA, Right coronary artery; LCA, Left coronary artery; LAD, Left anterior descending artery; CI, Confidence interval. IModel 1, unadjusted; ${ }^{*}$ Model 2, adjusted for age and sex.

vasculopathy ${ }^{2}$, retinal arteriolar changes in our study could reflect the degree of KD related inflammation and might even indicate the degree of coronary arterial damage. Estimates of all associations in our study were either attenuated or diminished after the application of coronary artery z-scores, which were calculated using BSA. As previous studies have reported strong associations between BMI and/or BSA with retinal vascular calibre among the paediatric population ${ }^{17,18}$, we speculated a possible BSA effect on the observed relationships between coronary artery structure and retinal microvasculature.

The strength of our study includes the use of quantitative measurements of both cardiac and retinal vascular measures, which is novel in a group of young children with new-onset of KD and the adoption of ECHO measures z-scores based on a large multi-race/ethnicity paediatric population. However, there are some potential limitations. First, the cross-sectional nature of the study design limits our interpretation on the temporal relationship of coronary artery parameters and retinal vascular parameters. Second, our findings may be biased by selection as patients were recruited only from a single paediatric hospital in Singapore, which may not be generalisable to other paediatric KD patients. Third, the small sample size limits the statistical power to detect other associations of retinal microvascular and cardiac parameters. Fourth, the adoption of z-scores referencing mainly American paediatric population instead of Asian paediatric subjects may have biased our observations. Lastly, our study did not correct for multiple comparisons and adjust for more confounders due to a small sample size.

Our study provided the proof-of-concept of significant associations between coronary arterial dilation and retinal arteriolar changes in a pilot setting. The retinal photography process was fast and non-invasive. For 


\begin{tabular}{|c|c|c|c|c|}
\hline \multirow{2}{*}{\multicolumn{2}{|c|}{$\begin{array}{l}\text { Retinal microvascular } \\
\text { measures }\end{array}$}} & \multicolumn{3}{|l|}{ Echo measures } \\
\hline & & \multirow{2}{*}{\begin{tabular}{|l|} 
RCA, z-score \\
Per unit $\uparrow$
\end{tabular}} & \multirow[t]{2}{*}{$\begin{array}{l}\text { LCA, z-score } \\
\text { Per unit } \uparrow\end{array}$} & \multirow[t]{2}{*}{$\begin{array}{l}\text { LAD, z-score } \\
\text { Per unit } \uparrow\end{array}$} \\
\hline \multicolumn{2}{|c|}{$\underline{\text { Retinal Arteriolar Diameter, } \mu \mathrm{m}}$} & & & \\
\hline Model 1 & $\begin{array}{l}\beta, 95 \% \mathrm{CI} \\
\text { P value }\end{array}$ & $\begin{array}{l}3.21(-9.96,16.38) \\
0.60\end{array}$ & $\begin{array}{l}2.30(-8.21,12.80) \\
0.63\end{array}$ & $\begin{array}{l}3.18(-10.15,16.51) \\
0.60\end{array}$ \\
\hline Model 2 & $\begin{array}{l}\beta, 95 \% \mathrm{CI} \\
\text { P value }\end{array}$ & $\begin{array}{l}3.68(-11.93,19.29) \\
0.60\end{array}$ & $\begin{array}{l}2.42(-11.11,15.96) \\
0.69\end{array}$ & $\begin{array}{l}3.59(-12.83,20.02) \\
0.62\end{array}$ \\
\hline \multicolumn{5}{|c|}{$\underline{\text { Retinal Venular Diameter, } \mu \mathrm{m}}$} \\
\hline Model 1 & $\begin{array}{l}\beta, 95 \% \mathrm{CI} \\
\mathrm{P} \text { value }\end{array}$ & $\begin{array}{l}-1.53(-18.00,14.94) \\
0.84\end{array}$ & $\begin{array}{l}4.93(-7.65,17.51) \\
0.40\end{array}$ & $\begin{array}{l}0.20(-16.49,16.90) \\
0.98\end{array}$ \\
\hline Model 2 & $\begin{array}{l}\beta, 95 \% \mathrm{CI} \\
\text { P value }\end{array}$ & $\begin{array}{l}-1.56(-21.25,18.13) \\
0.86\end{array}$ & $\begin{array}{l}5.16(-11.17,21.48) \\
0.48\end{array}$ & $\begin{array}{l}0.97(-19.73,21.66) \\
0.92\end{array}$ \\
\hline \multicolumn{5}{|c|}{ Retinal Arteriolar Fractal Dimension, Df } \\
\hline Model 1 & $\begin{array}{l}\beta, 95 \% \mathrm{CI} \\
\text { P value }\end{array}$ & $\begin{array}{l}0.05(-0.01,0.11) \\
0.09\end{array}$ & $\begin{array}{l}-0.40 \times 10^{-2}(-0.06,0.05) \\
0.86\end{array}$ & $\begin{array}{l}0.06\left(0.39 \times 10^{-3}, 0.11\right) \\
0.05\end{array}$ \\
\hline Model 2 & $\begin{array}{l}\beta, 95 \% \mathrm{CI} \\
\text { P value }\end{array}$ & $\begin{array}{l}0.05(-0.02,0.12) \\
0.15\end{array}$ & $\begin{array}{l}0.12 \times 10^{-2}(-0.70,0.07) \\
0.97\end{array}$ & $\begin{array}{l}0.06(-0.01,0.13) \\
0.09\end{array}$ \\
\hline \multicolumn{5}{|c|}{ Retinal Venular Fractal Dimension, Df } \\
\hline Model 1 & $\begin{array}{l}\beta, 95 \% \mathrm{CI} \\
\mathrm{P} \text { value }\end{array}$ & $\begin{array}{l}0.02(-0.04,0.09) \\
0.44\end{array}$ & $\begin{array}{l}-0.42 \times 10^{-2}(-0.06,0.05) \\
0.87\end{array}$ & $\begin{array}{l}0.02(-0.05,0.08) \\
0.61\end{array}$ \\
\hline Model 2 & $\begin{array}{l}\beta, 95 \% \mathrm{CI} \\
\text { P value }\end{array}$ & $\begin{array}{l}0.02(-0.05,0.09) \\
0.54\end{array}$ & $\begin{array}{l}0.76 \times 10^{-2}(-0.06,0.07) \\
0.79\end{array}$ & $\begin{array}{l}0.80 \times 10^{-2}(-0.07,0.09) \\
0.82\end{array}$ \\
\hline \multicolumn{5}{|c|}{ Retinal Arteriolar Branching Angle, degree } \\
\hline Model 1 & \begin{tabular}{|l|}
$\beta, 95 \% \mathrm{CI}$ \\
P value
\end{tabular} & $\begin{array}{l}4.22(-7.58,16.01) \\
0.44\end{array}$ & $\begin{array}{l}6.53(-1.84,14.90) \\
0.11\end{array}$ & $\begin{array}{l}3.09(-9.04,15.22) \\
0.58\end{array}$ \\
\hline Model 2 & $\begin{array}{l}\beta, 95 \% \mathrm{CI} \\
\text { P value }\end{array}$ & $\begin{array}{l}4.11(-8.56,16.78) \\
0.47\end{array}$ & $\begin{array}{l}5.66(-4.49,15.80) \\
0.23\end{array}$ & $\begin{array}{l}5.70(-7.17,18.57) \\
0.33\end{array}$ \\
\hline \multicolumn{5}{|c|}{ Retinal Venular Branching Angle, degree } \\
\hline Model 1 & $\begin{array}{l}\beta, 95 \% \mathrm{CI} \\
\text { P value }\end{array}$ & $\begin{array}{l}0.77(-13.68,15.22) \\
0.91\end{array}$ & $\begin{array}{l}0.37(-11.11,11.86) \\
0.94\end{array}$ & $\begin{array}{l}-2.89(-17.35,11.57) \\
0.66\end{array}$ \\
\hline Model 2 & $\begin{array}{l}\beta, 95 \% \mathrm{CI} \\
\text { P value }\end{array}$ & $\begin{array}{l}1.59(-15.17,18.34) \\
0.83\end{array}$ & $\begin{array}{l}-0.26(-14.71,14.19) \\
0.97\end{array}$ & $\begin{array}{l}-3.03(-20.47,14.41) \\
0.69\end{array}$ \\
\hline \multicolumn{5}{|c|}{$\underline{\text { Retinal Arteriolar Curvature Tortuosity, unit }}-\underline{-5}$} \\
\hline Model 1 & $\begin{array}{l}\beta, 95 \% \mathrm{CI} \\
\text { P value }\end{array}$ & $\begin{array}{l}0.83(-3.01,4.67) \\
0.64\end{array}$ & $\begin{array}{l}1.51(-1.37,4.38) \\
0.27\end{array}$ & $\begin{array}{l}1.20(-2.63,5.03) \\
0.50\end{array}$ \\
\hline Model 2 & $\begin{array}{l}\beta, 95 \% \mathrm{CI} \\
\text { P value }\end{array}$ & $\begin{array}{l}0.40(-3.31,4.11) \\
0.81\end{array}$ & $\begin{array}{l}1.78(-1.00,4.56) \\
0.18\end{array}$ & $\begin{array}{l}1.66(-1.96,5.28) \\
0.32\end{array}$ \\
\hline \multicolumn{5}{|c|}{$\underline{\text { Retinal Venular Curvature Tortuosity, unit }}=\underline{-5}$} \\
\hline Model 1 & $\begin{array}{l}\beta, 95 \% \mathrm{CI} \\
\text { P value }\end{array}$ & $\begin{array}{l}0.42(-2.36,3.20) \\
0.74\end{array}$ & $\begin{array}{l}0.55(-1.63,2.74) \\
0.58\end{array}$ & $\begin{array}{l}-1.28(-3.94,1.38) \\
0.30\end{array}$ \\
\hline Model 2 & $\begin{array}{l}\beta, 95 \% \mathrm{CI} \\
\text { P value }\end{array}$ & $\begin{array}{l}0.44(-2.17,3.05) \\
0.70\end{array}$ & $\begin{array}{l}-0.14(-2.40,2.12) \\
0.89\end{array}$ & $\begin{array}{l}-0.63(-3.34,2.08) \\
0.60\end{array}$ \\
\hline
\end{tabular}

Table 3. Association between coronary artery parameters z-scores and retinal vessel parameters in patients with new onset of Kawasaki disease. Abbreviations: RCA, Right coronary artery; LCA, Left coronary artery;

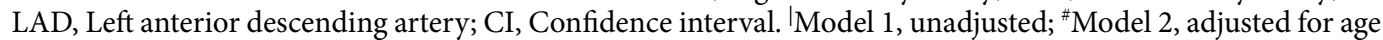
and sex.

paediatric patients between 3-4 years old, the retinal photography, on average, took 15 minutes. For older or more cooperative paediatric patients, this process was shortened to $<10$ minutes. Future studies with larger samples and longer follow-up periods are warranted to further explore the potential screening values of retinal microvasculature in children, in terms of detecting coronary arterial involvements in the acute phase of new-onset KD. Further studies are also warranted to obtain post-treatment changes in ECHO and retinal parameters in order to determine the duration and direction of both changes in coronary and retinal vessels. Additionally, future studies should explore whether the magnitude of vessel changes are more pronounced among children with KD who have abnormal z-scores.

\section{Data availability}

The datasets generated during and/or analysed during the current study are not publicly available to minimise the possibility of unintentionally sharing information that can be used to re-identify private information, but are available from the corresponding author on reasonable request.

Received: 18 December 2018; Accepted: 24 October 2019;

Published online: 13 November 2019 


\section{References}

1. Burns, J. C. \& Glode, M. P. Kawasaki syndrome. Lancet (London, England) 364, 533-544, https://doi.org/10.1016/s01406736(04)16814-1 (2004).

2. McCrindle, B. W. et al. Diagnosis, Treatment, and Long-Term Management of Kawasaki Disease: A Scientific Statement for Health Professionals From the American Heart Association. Circulation 135, e927-e999, https://doi.org/10.1161/cir.0000000000000484 (2017).

3. Varvarigou, A. A. Intrauterine growth restriction as a potential risk factor for disease onset in adulthood. Journal of pediatric endocrinology \& metabolism: JPEM 23, 215-224 (2010).

4. Hubbard, L. D. et al. Methods for evaluation of retinal microvascular abnormalities associated with hypertension/sclerosis in the Atherosclerosis Risk in Communities Study. Ophthalmology 106, 2269-2280 (1999).

5. Chen, K. Y. et al. Evidence of Microvascular Changes in the Retina following Kawasaki Disease. Scientific reports 7, 40513, https:// doi.org/10.1038/srep40513 (2017).

6. Lopez, L. et al. Relationship of Echocardiographic Z Scores Adjusted for Body Surface Area to Age, Sex, Race, and Ethnicity: The Pediatric Heart Network Normal Echocardiogram Database. Circulation. Cardiovascular imaging 10, https://doi.org/10.1161/ circimaging.117.006979 (2017).

7. Haycock, G. B., Schwartz, G. J. \& Wisotsky, D. H. Geometric method for measuring body surface area: a height-weight formula validated in infants, children, and adults. The Journal of pediatrics 93, 62-66 (1978).

8. The fourth report on the diagnosis, evaluation, and treatment of high blood pressure in children and adolescents. Pediatrics 114, 555-576 (2004)

9. Centers for Disease Control and Prevention. Percentile Data Files with LMS Values. Available from https://www.cdc.gov/ growthcharts/percentile_data_files.htm (2010).

10. Cheung, C. Y. et al. A new method to measure peripheral retinal vascular caliber over an extended area. Microcirculation (New York, N.Y.: 1994) 17, 495-503, https://doi.org/10.1111/j.1549-8719.2010.00048.x (2010).

11. Liew, G. et al. The retinal vasculature as a fractal: methodology, reliability, and relationship to blood pressure. Ophthalmology 115, 1951-1956, https://doi.org/10.1016/j.ophtha.2008.05.029 (2008).

12. Cheung, C. Y. et al. Retinal vascular tortuosity, blood pressure, and cardiovascular risk factors. Ophthalmology 118, 812-818, https:// doi.org/10.1016/j.ophtha.2010.08.045 (2011).

13. Tomita, Y. et al. Long-term in vivo investigation of mouse cerebral microcirculation by fluorescence confocal microscopy in the area of focal ischemia. Journal of cerebral blood flow and metabolism: official journal of the International Society of Cerebral Blood Flow and Metabolism 25, 858-867, https://doi.org/10.1038/sj.jcbfm.9600077 (2005).

14. Yamakawa, K., Bhutto, I. A., Lu, Z., Watanabe, Y. \& Amemiya, T. Retinal vascular changes in rats with inherited hypercholesterolemia-corrosion cast demonstration. Current eye research 22, 258-265 (2001).

15. Witt, N. et al. Abnormalities of retinal microvascular structure and risk of mortality from ischemic heart disease and stroke. Hypertension (Dallas, Tex.: 1979) 47, 975-981, https://doi.org/10.1161/01.HYP.0000216717.72048.6c (2006).

16. Owen, C. G. et al. Retinal arteriolar tortuosity and cardiovascular risk factors in a multi-ethnic population study of 10-year-old children; the Child Heart and Health Study in England (CHASE). Arteriosclerosis, thrombosis, and vascular biology 31, 1933-1938, https://doi.org/10.1161/atvbaha.111.225219 (2011).

17. Cheung, N. et al. Distribution and associations of retinal vascular caliber with ethnicity, gender, and birth parameters in young children. Investigative ophthalmology \& visual science 48, 1018-1024, https://doi.org/10.1167/iovs.06-0978 (2007).

18. Taylor, B. et al. Body mass index and its effects on retinal vessel diameter in 6-year-old children. International journal of obesity (2005) 31, 1527-1533, https://doi.org/10.1038/sj.ijo.0803674 (2007).

\section{Acknowledgements}

We thank all mothers for participating in this study, and study team for running this study. This study entitled as "Exploring the potential diagnostic value of retinal microvascular morphology in pediatric Kawasaki Disease" is funded by Khoo Pilot Award, Duke-NUS Medical School (Duke-NUS-KP/2015/0018). Dr. Ling-Jun Li is funded by Singapore National Medical Council Transition Award (NMRC/TA/0027/2014) and NMRC centre grant (NMRC/CG/C008A/2017_KKH).

\section{Author contributions}

E.J.L. performed data analyses and wrote up the whole manuscript. I.M.A. reviewed the manuscript, contributed to the discussion and edition of the manuscript. J.C. designed the study, provided the data and reviewed the manuscript. W.T.Y. interpreted the data, reviewed and edited the manuscript. L.-J.L. designed the study, interpreted the data, reviewed and edited the manuscript.

\section{Competing interests}

The authors declare no competing interests.

\section{Additional information \\ Supplementary information is available for this paper at https://doi.org/10.1038/s41598-019-53220-3.}

Correspondence and requests for materials should be addressed to L.-J.L.

Reprints and permissions information is available at www.nature.com/reprints.

Publisher's note Springer Nature remains neutral with regard to jurisdictional claims in published maps and institutional affiliations.

(c) (i) Open Access This article is licensed under a Creative Commons Attribution 4.0 International License, which permits use, sharing, adaptation, distribution and reproduction in any medium or format, as long as you give appropriate credit to the original author(s) and the source, provide a link to the Creative Commons license, and indicate if changes were made. The images or other third party material in this article are included in the article's Creative Commons license, unless indicated otherwise in a credit line to the material. If material is not included in the article's Creative Commons license and your intended use is not permitted by statutory regulation or exceeds the permitted use, you will need to obtain permission directly from the copyright holder. To view a copy of this license, visit http://creativecommons.org/licenses/by/4.0/.

(c) The Author(s) 2019 